\title{
GLOBAL DYNAMICS OF A LOTKA-VOLTERRA MODEL WITH TWO PREDATORS COMPETING FOR ONE PREY
}

\author{
JAUME LLIBRE AND DONGMEI XIAO
}

\begin{abstract}
In this paper we study the global dynamics of 3-dimensional predator prey Lotka-Volterra systems, which describes two predators competing for food or shared one resource. From theoretical analysis on all parameters of this system, we show that if the resource for prey is limited, then there exist some values of parameters such that two predators and one prey coexist and their population are asymptotic to steady states. Otherwise, at least one of two predator species is extinct. On the other hand, if the resource for prey is unlimited, then there are the complete classification of parameters values such that the system has two possible global dynamics. Either every solution of the system is asymptotic to a closed orbit, or to the equilibrium in the invariant coordinate plane, or every solution of the system is a periodic orbit except the equilibrium in the positive octant of $\mathbb{R}^{3}$. This implies that the principle of competitive exclusion holds for some values of parameters of the Lotka-Volterra system, and it does not hold for the other values of parameters of the Lotka-Volterra system. Hence, there are only two coexistence styles for all three species: periodic oscillation or steady states, which depends on the resource for prey. The results have an importance biological implication in pest control.
\end{abstract}

\section{INTRODUCTION}

The study of the dynamics of predator-prey systems was originated in the works of Lotka [17] and Volterra [25] who considered a model for one predator and one single prey in a constant and uniform environment, known now as the standard Lotka-Volterra model. In this model the populations of predator and prey permanently oscillate for almost all positive initial conditions. In the same work, Volterra also argued that the coexistence of two or more predators competing for fewer prey resources is impossible, this claim was called the principle of competitive exclusion (see [1], [7], [19]).

Hsu and Hubbell in [9] studied 4-dimensional Lotka-Volterra models with two competing predators sharing two prey species under the assumption that prey species were capable of self-reproduction and regenerated logistically in the absence of consumption, and obtained some conditions under which the competing predators survive or die out. Their results revealed that the principle of competitive exclusion was true for some of parameters values in the Lotka-Volterra model with two competing predators sharing two prey species. Note that the predator functional response to the prey density is linear in the Lotka-Volterra model. When the predator functional response to the prey density is nonlinear, the principle of

1991 Mathematics Subject Classification. 37N25, 34C12, 34C28, 37G20.

Key words and phrases. Predator-prey, Lotka-Volterra model, global dynamics, extinction, coexistence. 
competitive exclusion was re-examined by Koch in [13] via numerical simulation, which showed that the coexistence of two predators competing for a single prey species was in fact possible when the predator functional response to the prey density was assumed according to the Michaelis-Menten kinetics, and such coexistence occurred along what appeared to be a periodic orbit in the positive octant of $\mathbb{R}^{3}$ rather than an equilibrium.

Hsu, Hubbell and Waltman in $[10,11]$ studied the competition problem of the two predators for a single prey model. By combining theoretical analysis with numerical simulations, they obtained the parameter range of the validity of the principle of competitive exclusion, and provided a wide range of parameter values for the coexistence of two predators numerically. Following these numerical observations, there have been several important theoretical developments in justifying the coexistence for two predators competing for a single prey species model with nonlinear functional responses. In which bifurcation techniques were applied to the model for obtaining a stable periodic orbit in the positive octant of $\mathbb{R}^{3}$. This stable periodic orbit bifurcates from a 2-dimensional predator-prey limit cycle in the plane, which implies the coexistence of two competing predators (see [3], [12], [22], [23] and their references therein). Muratori and Rinald in [20] first considered the problem by singular perturbation arguments. Using dynamical systems techniques and the geometric singular perturbation theory, Liu, Xiao and Yi in [16] gave precise conditions which guarantee the coexistence of two predators for the model via rigorously analysis.

It is well known that resource competition is common in nature and society. Global dynamics of the resource competitive model is important to understand the mechanism of natural selection: the principle of competitive exclusion and the coexistence of competing species. There have been some excellent works such as [15], [26] and so on.

The aim of this paper is to study the global dynamics of 3-dimensional LotkaVolterra models with two predators competing for a single prey species in a constant and uniform environment. It is assumed that the two predator species compete purely exploitatively with no interference between rivals, the growth rate of the prey species is logistic or linear in the absence of predation, respectively, and the predator's functional response is linear. Then the model is a system of ordinary differential equations of the form

$$
\begin{aligned}
& \frac{d S(t)}{d t}=S(t)\left(r_{3}-\frac{1}{K} S(t)-b_{1} x_{1}(t)-b_{2} x_{2}(t)\right), \\
& \frac{d x_{1}(t)}{d t}=x_{1}(t)\left(-r_{1}+a_{1} S(t)\right), \\
& \frac{d x_{2}(t)}{d t}=x_{2}(t)\left(-r_{2}+a_{2} S(t)\right),
\end{aligned}
$$

where $x_{i}(t)$ for $i=1,2$ represents the population density of the $i$-th predator at time $t, S(t)$ represents the population density of the prey at time $t, r_{3}>0$ is the intrinsic rate of growth of the prey, $K>0$ is the carrying capacity of the prey, which describes the richness of resources for prey. If $K=\infty$, then prey can increase unlimited, which implies that the growth rate of the prey species is linear in the absence of predation; $b_{i}>0$ is the effect of the $i$-th predation on the prey, $r_{i}>0$ is the natural death rate of the $i$-th predator in the absence of prey, $a_{i}$ is the effciency 
and propagation rate of the $i$-th predator in the presence of prey. It is clear that $x_{i}(t) \geq 0$ and $S(t) \geq 0$. Hence, system (1) is considered only in the closed positive octant $\mathbb{R}_{+}^{3}$, of course here $\mathbb{R}_{+}=[0, \infty)$. For simplicity, we denote the open positive octant by $\operatorname{Int}\left(\mathbb{R}_{+}^{3}\right)$.

We completely characterize the qualitative behavior of system (1) in two cases: the limited resource for prey (i.e. $K$ is bounded) and the unlimited resource for prey (i.e. $K=\infty$ ). It is shown that as $K$ is bounded, there exist some values of parameters of system (1) such that two predators and one prey coexist and their population are asymptotic to steady states. Otherwise one of two predators species is extinct. On the other hand, if $K=\infty$, then there are only two possible global dynamics for the system. Either the system has a global attractor which is a boundary equilibrium, or every solution of the system is a periodic orbit in the positive octant of $\mathbb{R}^{3}$. These results imply that the principle of competitive exclusion hold for some values of parameters of the Lotka-Volterra system, and it does not hold for the other values of parameters of the Lotka-Volterra system. And there are only two coexistence styles for all three species of system (1): periodic oscillation or steady states, which depends on the resource for prey.

This paper is organized as follows. In section 2 we study the global dynamics of the two predators-one prey system as $K$ is a bounded parameter. In section 3 we study the global dynamics of the two predators-one prey system when $K=+\infty$. The paper ends with a brief discussion.

\section{Global dynamics of System (1) With limited Resource for PRey}

In this section we consider system (1) with the bounded $K$ in $\mathbb{R}_{+}^{3}$. We first study the existence and topological classification of finite equilibria and equilibria at infinity for system (1), then we study the global dynamics of this system.

2.1. The existence and topological classification of equilibria. It is clear that system (1) has an equilibrium at the origin $O(0,0,0)$, and an equilibrium at the point $E_{0}\left(r_{3} K, 0,0\right)$ for all the values of the parameters. Except the two boundary equilibria, system (1) may have other boundary equilibria and the positive equilibria in $\mathbb{R}_{+}^{3}$ for some values of parameters. Doing easy algebra calculations we obtain the following result.

Proposition 1. The following statements hold.

(i) System (1) has a boundary equilibrium $E_{1}\left(\frac{r_{1}}{a_{1}}, \frac{1}{b_{1}}\left(r_{3} K-\frac{r_{1}}{a_{1}}\right), 0\right)$ in $\mathbb{R}_{+}^{3}$ except the boundary equilibria $O$ and $E_{0}$ if $r_{3} K>\frac{r_{1}}{a_{1}}$; and system (1) has a boundary equilibrium $E_{2}\left(\frac{r_{2}}{a_{2}}, 0, \frac{1}{b_{2}}\left(r_{3} K-\frac{r_{2}}{a_{2}}\right)\right)$ in $\mathbb{R}_{+}^{3}$ except the boundary equilibria $O$ and $E_{0}$ if $r_{3} K>\frac{r_{2}}{a_{2}}$.

(ii) System (1) has positive equilibria $E_{+}\left(S, x_{1}, x_{2}\right)$ in $\operatorname{Int}\left(\mathbb{R}_{+}^{3}\right)$ if and only if $\frac{r_{1}}{a_{1}}=\frac{r_{2}}{a_{2}}$ and $r_{3} K>\frac{r_{1}}{a_{1}}$. And if system (1) has a positive equilibria in $\operatorname{Int}\left(\mathbb{R}_{+}^{3}\right)$, then system (1) has infinitely many positive equilibria $E_{+}\left(S, x_{1}, x_{2}\right)$ in $\operatorname{Int}\left(\mathbb{R}_{+}^{3}\right)$, which fill up a segment $L$ with endpoints at boundary equilibria $E_{1}$ and $E_{2}$, respectively in $\operatorname{Int}\left(\mathbb{R}_{+}^{3}\right)$, where

$$
L=\left\{\left(S, x_{1}, x_{2}\right): S=\frac{r_{1}}{a_{1}}, b_{1} x_{1}+b_{2} x_{2}=r_{3} K-\frac{r_{1}}{a_{1}}, x_{1} \geq 0, x_{2} \geq 0\right\} .
$$


We now study the local phase portraits of the boundary equilibria and positive equilibria. Since the Jacobian matrix $J\left(S, x_{1}, x_{2}\right)$ of system (1) at an equilibrium $\left(S, x_{1}, x_{2}\right)$ is

$$
J\left(S, x_{1}, x_{2}\right)=\left(\begin{array}{ccc}
r_{3}-\frac{2}{K} S-b_{1} x_{1}-b_{2} x_{2} & -b_{1} S & -b_{2} S \\
a_{1} x_{1} & -r_{1}+a_{1} S & 0 \\
a_{2} x_{2} & 0 & -r_{2}+a_{2} S
\end{array}\right),
$$

we obtain the following local phase portraits for the equilibria after some computations and the applications of standard results.

Proposition 2. The following statements hold.

(i) $O(0,0,0)$ is a saddle with a 2-dimensional stable manifold and an 1-dimensional unstable manifold for all parameters.

(ii) $E_{0}\left(r_{3} K, 0,0\right)$ has the following local phase portraits depending on the values of the parameters.

(ii.a) $E_{0}$ is a stable node if $r_{3} K<\min \left\{\frac{r_{1}}{a_{1}}, \frac{r_{2}}{a_{2}}\right\}$;

(ii.b) $E_{0}$ is saddle with a 2-dimensional stable manifold and an 1-dimensional unstable manifold if $\min \left\{\frac{r_{1}}{a_{1}}, \frac{r_{2}}{a_{2}}\right\}<r_{3} K<\max \left\{\frac{r_{1}}{a_{1}}, \frac{r_{2}}{a_{2}}\right\}$;

(ii.c) $E_{0}$ is saddle with a 1-dimensional stable manifold and a 2-dimensional unstable manifold if $\max \left\{\frac{r_{1}}{a_{1}}, \frac{r_{2}}{a_{2}}\right\}<r_{3} K$;

(ii.d) $E_{0}$ is degenerated equilibrium if either $r_{3} K=\frac{r_{1}}{a_{1}}$ or $r_{3} K=\frac{r_{2}}{a_{2}}$.

(iii) $E_{1}\left(\frac{r_{1}}{a_{1}}, \frac{1}{b_{1}}\left(r_{3} K-\frac{r_{1}}{a_{1}}\right), 0\right)$ has the following local phase portraits depending on the values of the parameters.

(iii.a) $E_{1}$ is a stable node if $\frac{r_{1}}{a_{1}}<r_{3} K \leq \frac{r_{1}+\sqrt{r_{1}^{2}+r_{1} r_{3}}}{2 a_{1}}$ and $\frac{r_{1}}{a_{1}}<\frac{r_{2}}{a_{2}}$;

(iii.b) $E_{1}$ is saddle with a 2-dimensional stable manifold and an 1-dimensional unstable manifold if $\frac{r_{1}}{a_{1}}<r_{3} K \leq \frac{r_{1}+\sqrt{r_{1}^{2}+r_{1} r_{3}}}{2 a_{1}}$ and $\frac{r_{1}}{a_{1}}>\frac{r_{2}}{a_{2}}$;

(iii.c) $E_{1}$ is stable focus if $r_{3} K>\frac{r_{1}+\sqrt{r_{1}^{2}+r_{1} r_{3}}}{2 a_{1}}$ and $\frac{r_{1}}{a_{1}}<\frac{r_{2}}{a_{2}}$;

(iii.d) $E_{1}$ is a saddle-focus with a 2-dimensional stable manifold and an 1dimensional unstable manifold if $r_{3} K>\frac{r_{1}+\sqrt{r_{1}^{2}+r_{1} r_{3}}}{2 a_{1}}$ and $\frac{r_{1}}{a_{1}}>\frac{r_{2}}{a_{2}}$;

(iii.e) $E_{1}$ is a degenerated equilibrium if $\frac{r_{1}}{a_{1}}<r_{3} K$ and $\frac{r_{1}}{a_{1}}=\frac{r_{2}}{a_{2}}$.

(iv) $E_{2}\left(\frac{r_{2}}{a_{2}}, 0, \frac{1}{b_{2}}\left(r_{3} K-\frac{r_{2}}{a_{2}}\right)\right)$ has the following local phase portraits depending on the values of the parameters.

(iv.a) $E_{2}$ is a stable node if $\frac{r_{2}}{a_{2}}<r_{3} K \leq \frac{r_{2}+\sqrt{r_{2}^{2}+r_{2} r_{3}}}{2 a_{2}}$ and $\frac{r_{1}}{a_{1}}>\frac{r_{2}}{a_{2}}$;

(iv.b) $E_{2}$ is saddle with a 2-dimensional stable manifold and an 1-dimensional unstable manifold if $\frac{r_{2}}{a_{2}}<r_{3} K \leq \frac{r_{2}+\sqrt{r_{2}^{2}+r_{2} r_{3}}}{2 a_{2}}$ and $\frac{r_{1}}{a_{1}}<\frac{r_{2}}{a_{2}}$;

(iv.c) $E_{2}$ is stable focus if $r_{3} K>\frac{r_{2}+\sqrt{r_{2}^{2}+r_{2} r_{3}}}{2 a_{2}}$ and $\frac{r_{1}}{a_{1}}>\frac{r_{2}}{a_{2}}$;

(iv.d) $E_{2}$ is a saddle-focus with a 2-dimensional stable manifold and an 1dimensional unstable manifold if $r_{3} K>\frac{r_{2}+\sqrt{r_{2}^{2}+r_{2} r_{3}}}{2 a_{2}}$ and $\frac{r_{1}}{a_{1}}<\frac{r_{2}}{a_{2}}$;

(iv.e) $E_{2}$ is a degenerated equilibrium if $\frac{r_{2}}{a_{2}}<r_{3} K$ and $\frac{r_{1}}{a_{1}}=\frac{r_{2}}{a_{2}}$.

(v) Each positive equilibrium $E_{+}$is a degenerated equilibrium with a 2-dimensional stable manifold and an 1-dimensional center manifold filled by equilibria.

It is clear that system (1) has three invariant planes $S=0, x_{1}=0$ and $x_{2}=0$, respectively. Our objective is to study the global dynamics of system (1) in $\mathbb{R}_{+}^{3}$. 
However, it is not clear whether or not all solutions of system (1) with positive initial conditions are bounded in forward time. So we must study the equilibria of system (1) at infinity and we shall use the Poincaré compactification of $\mathbb{R}_{+}^{3}$ for doing this study. Note that system (1) defines a polynomial vector field in $\mathbb{R}^{3}$.

Using the Poincaré compactification in $\mathbb{R}^{3}$ (see for more details [6]), we consider the unit sphere $\mathbb{S}^{3}=\left\{\left(y_{1}, y_{2}, y_{3}, y_{4}\right) \in \mathbb{R}^{4}: \sum_{i=1}^{4} y_{i}^{2}=1\right\}$ in $\mathbb{R}^{4}$. Its equator is $\mathbb{S}^{2}=\left\{\left(y_{1}, y_{2}, y_{3}, y_{4}\right) \in \mathbb{S}^{3}: y_{4}=0\right\}$. We identify $\mathbb{R}^{3}$ with the tangent hyperplane at the north pole of the sphere $\mathbb{S}^{3}$, i.e. at the point $(0,0,0,1)$. Doing central projection of this hyperplane over the sphere $\mathbb{S}^{3}, \mathbb{R}^{3}$ is identified with the open north hemisphere of $\mathbb{S}^{3}$. Clearly $\mathbb{S}^{2}$ is the boundary of this open north hemisphere. The open north hemisphere together with its boundary $\mathbb{S}^{2}$ is topologically a closed ball $B$, whose interior is identified with $\mathbb{R}^{3}$ and whose boundary is identified with the infinity of $\mathbb{R}^{3}$. Every point of $\mathbb{S}^{2}$ corresponds with one direction for reaching the infinity.

Summarizing system (1) in $\mathbb{R}^{3}$ can be extended analytically to a vector field in the closed ball $B$, called the Poincaré compactification of system (1). The infinity $\mathbb{S}^{2}$ is invariant under the flow of the extended vector field. The flow on $\mathbb{S}^{2}$ is studied taking the local charts defined in [6].

In the local chart $U_{1}$ with coordinates

$$
S=\frac{1}{z_{3}}, x_{1}=\frac{z_{1}}{z_{3}}, x_{2}=\frac{z_{2}}{z_{3}},
$$

system (1) becomes

$$
\begin{aligned}
& \frac{d z_{1}(t)}{d t}=a_{1} z_{1}+\frac{z_{1}}{K}+b_{1} z_{1}^{2}+b_{2} z_{1} z_{2}-r_{1} z_{1} z_{3}-r_{3} z_{1} z_{3}, \\
& \frac{d z_{2}(t)}{d t}=a_{2} z_{2}+\frac{z_{2}}{K}+b_{1} z_{1} z_{2}+b_{2} z_{2}^{2}-r_{2} z_{2} z_{3}-r_{3} z_{2} z_{3}, \\
& \frac{d z_{3}(t)}{d t}=\frac{z_{3}}{K}+b_{1} z_{1} z_{3}+b_{2} z_{2} z_{3}-r_{3} z_{3}^{2} .
\end{aligned}
$$

The infinity of $\mathbb{R}^{3}$ in the local chart $U_{1}$ corresponds to the invariant plane $z_{3}=0$ and the infinity of $\mathbb{R}_{+}^{3}$ in the local chart $U_{1}$ corresponds to the invariant plane $z_{3}=0$ with $z_{1} \geq 0$ and $z_{2} \geq 0$. Hence, system (2) in the local chart $U_{1}$ has a unique equilibrium at infinity $O_{i}(0,0,0)$. The linear part of system $(2)$ at $O_{i}(0,0,0)$ has eigenvalues $a_{1}+\frac{1}{K}, a_{2}+\frac{1}{K}$ and $\frac{1}{K}$. This implies that equilibrium $O_{i}(0,0,0)$ is an unstable node.

Since the local chart $U_{1}$ does not cover the plane $S=0$ at the infinity of $\mathbb{R}^{3}$, we further consider system (1) in the local chart $U_{2}$ with coordinates

$$
S=\frac{u_{1}}{z_{3}}, x_{1}=\frac{1}{z_{3}}, x_{2}=\frac{u_{2}}{z_{3}},
$$

which becomes

$$
\begin{aligned}
& \frac{d u_{1}(t)}{d t}=-b_{1} u_{1}-a_{1} u_{1}^{2}-\frac{u_{1}^{2}}{K}-b_{2} u_{1} u_{2}+r_{1} u_{1} z_{3}+r_{3} u_{1} z_{3}, \\
& \frac{d u_{2}(t)}{d t}=-a_{1} u_{1} u_{2}+a_{2} u_{1} u_{2}+r_{1} u_{2} z_{3}-r_{2} u_{2} z_{3}, \\
& \frac{d z_{3}(t)}{d t}=-a_{1} u_{1} z_{3}+r_{1} z_{3}^{2} .
\end{aligned}
$$


Note that all the points on $\mathbb{S}^{2}$ at infinity in the coordinates of all local chart have $z_{3}=0$. We are interested only in the equilibria with $u_{1}=0$ and $z_{3}=0$ of system (3), which corresponds to the infinity of $\mathbb{R}^{3}$ in the plane $S=0$ for system (1). If we further restrict at the infinity of $\mathbb{R}_{+}^{3}$, then system (3) has infinitely many equilibria $E_{I}\left(0, u_{2}, 0\right)$ with $u_{2} \geq 0$ at infinity, which fill up the quarter of a circle on $\mathbb{S}^{2}$. At each equilibrium $E_{I}\left(0, u_{2}, 0\right)$, system (3) has a double zero eigenvalue and a negative eigenvalue $-\left(b_{1}+b_{2} u_{2}\right)$ with the eigenvector $\left(\frac{b_{1}+b_{2} u_{2}}{\left(a_{1}-a_{2}\right) u_{2}}, 1,0\right)^{T}$ in its infinite direction when $a_{1} \neq a_{2}$. The eigenvector associated with the negative eigenvalue when $a_{1}=a_{2}$ is $(1,0,0)^{T}$ in its infinite direction. Obviously, the remaining eigenvalues 0 correspond to eigenvectors going outside the infinity.

Applying the normal hyperbolicity theory (see [8]) first to the local chart $U_{2}$ and after to the invariant plane $S=0$, we obtain that every equilibrium $E_{I}\left(0, u_{2}, 0\right)$ has an 1-dimensional stable manifold living at infinity (i.e. in $\mathbb{S}^{2}$ ) and two 1dimensional unstable manifold living in the plane $S=0$.

On the other hand, it is easy to check that both local charts $U_{1}$ and $U_{2}$ together have covered all equilibria at the infinity of system (1) in $\mathbb{R}_{+}^{3}$ except the equilibrium point localized at the endpoint of the $x_{2}$-axis in the infinity, but this equilibrium forms part of the line filled of equilibria at infinity. Hence, we can summarize the above analysis as follows.

Proposition 3. The following two statements hold.

(i) System (1) restricted to the compactification of the octant $\mathbb{R}_{+}^{3}$ has an isolated equilibrium $O_{i}$ at infinity which is the endpoint of the invariant positive $S$-axis, and has a line filled of equilibria $E_{I}$ at infinity which is the end of the invariant plane $S=0$ intersection with the compactification of the octant $\mathbb{R}_{+}^{3}$. This line of equilibria fills the quarter of the maximal circle at infinity with endpoints the endpoints of the invariant positive $x_{1}$-axis and $x_{2}$-axis.

(ii) Furthermore $O_{i}$ is an unstable node and each degenerate equilibria $E_{I}$ has 1-dimensional stable manifold living at infinity and two 1-dimensional unstable one living in the invariant plane $S=0$, which is the central manifold of the point $E_{I}$. Moreover, the behavior of the flow near these two 1-dimensional manifolds in the finite positive octant is like a hyperbolic sector. The phase portrait of these equilibria on $\mathbb{S}^{2}$ is shown in Figure 1.

Proof. The analysis done before the statement of the proposition has shown the existence and the local phase portraits of these equilibria at infinity. We only need to study the dynamics of system (1) on the center manifold of each equilibria $E_{I}$.

Since the positive quadrant of the $x_{1} x_{2}$-plane is invariant by the flow of system (1) and it is tangent to the center subspaces of each equilibria $E_{I}$, the $x_{1} x_{2}$-plane is the center manifold at each equilibria $E_{I}$.

We shall study the phase portrait of system (1) on the positive quadrant of the invariant $x_{1} x_{2}$-plane. On this plane the flow is determined by

$$
\begin{aligned}
& \frac{d x_{1}(t)}{d t}=-r_{1} x_{1}(t), \\
& \frac{d x_{2}(t)}{d t}=-r_{2} x_{2}(t),
\end{aligned}
$$


where $r_{1}$ and $r_{2}$ are positive constants.

It is clear that all solutions of system (4) on the $x_{1} x_{2}$-plane except the equilibria at infinity will approach to the origin $(0,0,0)$ in forward time. Hence, $E_{I}$ has two 1-dimensional unstable manifold on its center manifold $x_{1} x_{2}$-plane. Note that the 1-dimensional stable manifold of $E_{I}$ is on $\mathbb{S}^{2}$. Hence, the phase portrait in the finite part and near each equilibria $E_{I}$ looks like a saddle. And the isolated equilibrium $O_{i}$ is an unstable node. Thus, we can sketch the phase portraits near all equilibria at infinity of system (4) on $\mathbb{S}^{2}$ and on $S=0$ in Figure 1.

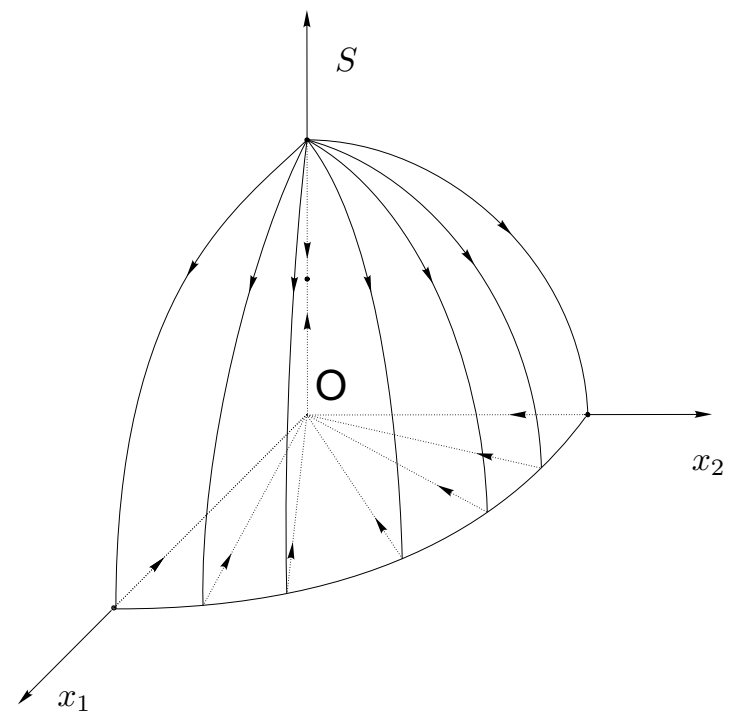

FIGURE 1. The phase portrait of system (1) near all equilibria at infinite on $\mathbb{S}^{2}$.

In the following, we analytically establish that all solutions of system (1) with positive initial conditions are bounded in forward time. This shows that system (1) is "well behaved" as one intuits from the biological problem.

Theorem 4. All orbits of system (1) with positive initial conditions are positive and bounded.

Proof. Since the flow of system (1) is invariant in the three coordinate planes of $\mathbb{R}_{+}^{3}$, all trajectories of system (1) with positive initial conditions remains in $\mathbb{R}_{+}^{3}$. From Proposition 3, we know that all equilibria at infinity of system (1) in its finite direction are repeller. Therefore, all trajectories of system (1) with positive initial conditions cannot reach the infinity. This implies that every trajectory is bounded.

2.2. Global dynamics. First we show that system (1) allows the coexistence of two competing predators for some values of parameters, which implies that the coexistence of two predators competing for a single prey species is possible when the predator functional response to the prey density is linear. And for some other values of parameters, both two competing predators go extinction and only prey survives. 
Theorem 5. If $\frac{r_{1}}{a_{1}}=\frac{r_{2}}{a_{2}}$, then system (1) has a first integral $F\left(S, x_{1}, x_{2}\right)=c$, where $F\left(S, x_{1}, x_{2}\right)=x_{1}^{a_{2}} x_{2}^{-a_{1}}$ and $c$ is any a constant. Furthermore

(i) if $r_{3} K>\frac{r_{1}}{a_{1}}$, then on the invariant surface $F\left(S, x_{1}, x_{2}\right)=c$ with $c>0$ in $\operatorname{Int}\left(\mathbb{R}_{+}{ }^{3}\right)$, system (1) has a unique positive equilibrium $E_{+}\left(\frac{r_{1}}{a_{1}},\left(c x_{2}^{a_{1}}\right)^{\frac{1}{a_{2}}}, x_{2}\right)$, which is a global attractor on $\left\{F\left(S, x_{1}, x_{2}\right)=c\right\} \cap \operatorname{Int}\left(\mathbb{R}_{+}{ }^{3}\right)$;

(ii) if $r_{3} K \leq \frac{r_{1}}{a_{1}}$, then on the invariant surface $F\left(S, x_{1}, x_{2}\right)=c$ with $c>0$ in $\operatorname{Int}\left(\mathbb{R}_{+}{ }^{3}\right)$, system (1) has not any equilibria and all orbits of (1) approach to a boundary point $\left(r_{3} K, 0,0\right)$ of this invariant surface in forward time.

Proof. Since the function $F\left(S, x_{1}, x_{2}\right)$ satisfies

$$
\left.\frac{d F\left(S, x_{1}, x_{2}\right)}{d t}\right|_{(1)}=a_{1} a_{2}\left(\frac{r_{2}}{a_{2}}-\frac{r_{1}}{a_{1}}\right) F\left(S, x_{1}, x_{2}\right),
$$

$\left.\frac{d F\left(S, x_{1}, x_{2}\right)}{d t}\right|_{(1)}=0$ as $\frac{r_{1}}{a_{1}}=\frac{r_{2}}{a_{2}}$. Hence, $F\left(S, x_{1}, x_{2}\right)$ is a first integral of system (1) by definition of the first integral.

Let

$$
F\left(S, x_{1}, x_{2}\right)=c, c>0 .
$$

Then the invariant surface, $F\left(S, x_{1}, x_{2}\right)=c$, of system (1) foliates Int $\mathbb{R}_{+}^{3}$. Obviously, the intersection of the invariant surface $F\left(S, x_{1}, x_{2}\right)=c$ and the invariant $x_{1} x_{2}$-plane is an orbit of system (1). And the intersection of the invariant surface and a given plane $S=\alpha$ with $\alpha>0$ in $\operatorname{Int} \mathbb{R}_{+}^{3}$ is a curve. It is easy to check that this curve is monotone function of $x_{1}$ or $x_{2}$.

We first prove statement (i). If $\frac{r_{1}}{a_{1}}=\frac{r_{2}}{a_{2}}$ and $r_{3} K>\frac{r_{1}}{a_{1}}$, then from proposition 1 , we know that system (1) has infinitely many positive equilibria $E_{+}\left(S, x_{1}, x_{2}\right)$ which fill up a segment $L$,

$$
L=\left\{\left(S, x_{1}, x_{2}\right): S=\frac{r_{1}}{a_{1}}, b_{1} x_{1}+b_{2} x_{2}=r_{3} K-\frac{r_{1}}{a_{1}}, x_{1} \geq 0, x_{2} \geq 0\right\} .
$$

Therefore, the surface $F\left(S, x_{1}, x_{2}\right)=c$ must cross $L$ at exact one point on the plane $S=\frac{r_{1}}{a_{1}}$.

Note that the flow of system (1) is invariant on each foliation $F\left(S, x_{1}, x_{2}\right)=c$ with $c>0$. Thus, the global dynamics of system (1) in $\operatorname{Int} \mathbb{R}_{+}^{3}$ is equivalent to that of system (1) restricted on each foliation $F\left(S, x_{1}, x_{2}\right)=c$ of int $\mathbb{R}_{+}^{3}$.

In what follows, we consider the restricted system

$$
\begin{aligned}
& \frac{d S(t)}{d t}=S(t)\left(r_{3}-\frac{1}{K} S(t)-b_{1}\left(c x_{2}^{a_{1}}(t)\right)^{\frac{1}{a_{2}}}-b_{2} x_{2}(t)\right), \\
& \frac{d x_{2}(t)}{d t}=x_{2}(t)\left(-r_{2}+a_{2} S(t)\right) .
\end{aligned}
$$

From the above analysis, we know that system (5) has a unique positive equilibrium at $\left(\frac{r_{1}}{a_{1}}, x_{2}^{+}\right)$, where $x_{2}^{+}$is a unique positive solution of

$$
b_{1}\left(c x_{2}^{a_{1}}\right)^{\frac{1}{a_{2}}}+b_{2} x_{2}=r_{3}-\frac{r_{1}}{a_{1} K} .
$$

By computing the Jacobian matrix of system (5) at $\left(\frac{r_{1}}{a_{1}}, x_{2}^{+}\right)$, we have that its two eigenvalues have negative real parts. Hence, the equilibrium is local asymptotically stable on the foliation. 
By Theorem 4, we know that every solution of system (5) is positive and bounded in the foliation. We next prove that the positive equilibrium at $\left(\frac{r_{1}}{a_{1}}, x_{2}^{+}\right)$is global stable in the foliation. Otherwise we assume that system (5) has a non-trivial periodic solution $\gamma(t)$ with period $T$ which is the first closed orbit near the positive equilibrium $\left(\frac{r_{1}}{a_{1}}, x_{2}^{+}\right)$,

$$
\gamma(t)=\left\{\left(S, x_{2}\right): S=S(t)>0, \quad x_{2}=x_{2}(t)>0,0 \leq t \leq T\right\} .
$$

Then by (5) we have

$$
\begin{aligned}
& 0=\ln \frac{S(T)}{S(0)}=\int_{0}^{T} \frac{d S(t)}{S(t)}=\int_{0}^{T}\left(r_{3}-\frac{1}{K} S(t)-b_{1}\left(c x_{2}^{a_{1}}(t)\right)^{\frac{1}{a_{2}}}-b_{2} x_{2}(t)\right) d t \\
& 0=\ln \frac{x_{2}(T)}{x_{2}(0)}=\int_{0}^{T} \frac{d x_{2}(t)}{x_{2}(t)}=\int_{0}^{T}\left(-r_{2}+a_{2} S(t)\right) d t
\end{aligned}
$$

where $\left(S(t), x_{2}(t)\right)$ is the non-trivial periodic solution $\gamma(t)$ of system (5).

In the following we consider the stability of $\gamma(t)$. We calculate the integral of divergence of vector field (5) along the periodic solution $\gamma(t)$.

$$
\begin{aligned}
\oint_{\gamma(t)} \operatorname{div}(5) d t & =\int_{0}^{T}\left(r_{3}-\frac{2}{K} S(t)-b_{1}\left(c x_{2}^{a_{1}}(t)\right)^{\frac{1}{a_{2}}}-b_{2} x_{2}(t)+\left(-r_{2}+a_{2} S(t)\right)\right) d t \\
& =\int_{0}^{T}-\frac{1}{K} S(t) d t<0
\end{aligned}
$$

by (6). Therefore, the periodic solution $\gamma(t)$ is stable. On the other hand, the unique equilibrium is local asymptotically stable on the foliation. This leads to a contradiction. Thus, the non-trivial periodic solution $\gamma(t)$ does not exist, which implies that the positive equilibrium at $\left(\frac{r_{1}}{a_{1}}, x_{2}^{+}\right)$is global stable in the foliation. Hence statement (i) holds.

In the following we consider the case that $\frac{r_{1}}{a_{1}}=\frac{r_{2}}{a_{2}}$ and $r_{3} K \leq \frac{r_{1}}{a_{1}}$. From Proposition 1 , we know that system (1) has not any equilibria in $\operatorname{Int} \mathbb{R}_{+}^{3}$. Hence, there do not exist periodic solutions of system (1) on the invariant surface $F\left(S, x_{1}, x_{2}\right)=c$.

It is clear that the global dynamics of system (1) in $\operatorname{Int} \mathbb{R}_{+}^{3}$ is equivalent to that of system (5) on the invariant surface. And all solutions of system (5) in Int $\mathbb{R}_{+}^{3}$ are bounded. On the boundary of the invariant surface $F\left(S, x_{1}, x_{2}\right)=c$, system (5) has two equilibria at $(0,0)$ and $\left(r_{3} K, 0\right)$, respectively. The equilibrium $\left(r_{3} K, 0\right)$ is a stable node and $(0,0)$ is a saddle with 1-dimensional stable manifold $\left\{F\left(S, x_{1}, x_{2}\right)=c\right\} \cap\left\{\left(S, x_{1}, x_{2}\right): S=0\right\}$ and 1-dimensional unstable manifold $\left\{\left(S, x_{1}, x_{2}\right): x_{1}=0, x_{2}=0,0<S<r_{3} K\right\}$. Hence, all solutions of system (5) with positive initial conditions approach to equilibrium $\left(r_{3} K, 0\right)$ in forward time. This proves statement (ii).

Theorem 5 has shown the dynamics of system (1) in $\operatorname{Int} \mathbb{R}_{+}^{3}$ if $\frac{r_{1}}{a_{1}}=\frac{r_{2}}{a_{2}}$. To obtain the global dynamics of system (1) in $\mathbb{R}_{+}^{3}$, we need to consider the dynamics of system (1) on the three invariant coordinate planes. Note that system (1) restricted on the invariant $x_{1} x_{2}$-plane has a unique equilibrium $(0,0)$ which is global stable for all parameters. We only consider the dynamics of system (1) on the invariant $S x_{1}$-plane and on the invariant $S x_{2}$-plane, respectively. 
On the invariant $S x_{1}$-plane, the restricted system of (1) is

$$
\begin{aligned}
& \frac{d S(t)}{d t}=S(t)\left(r_{3}-\frac{1}{K} S(t)-b_{1} x_{1}(t)\right), \\
& \frac{d x_{1}(t)}{d t}=x_{1}(t)\left(-r_{1}+a_{1} S(t)\right) .
\end{aligned}
$$

And on the invariant $S x_{2}$-plane, the restricted system of (1) is

$$
\begin{aligned}
& \frac{d S(t)}{d t}=S(t)\left(r_{3}-\frac{1}{K} S(t)-b_{2} x_{2}(t)\right), \\
& \frac{d x_{2}(t)}{d t}=x_{2}(t)\left(-r_{2}+a_{2} S(t)\right) .
\end{aligned}
$$

From the knowledge of the 2-dimensional Lotka-Volterra system, it follows easily the next result.

Proposition 6. The following two statements hold.

(i) If $\frac{r_{1}}{a_{1}}=\frac{r_{2}}{a_{2}}$ and $r_{3} K>\frac{r_{1}}{a_{1}}$, then system (7) and system (8) has three equilibria at $(0,0),\left(r_{3} K, 0\right)$ and $\left(\frac{r_{1}}{a_{1}}, \frac{1}{b_{1}}\left(r_{3}-\frac{r_{1}}{K a_{1}}\right)\right)$, where $(0,0)$ and $\left(r_{3} K, 0\right)$ are saddles, and $\left(\frac{r_{1}}{a_{1}}, \frac{1}{b_{1}}\left(r_{3}-\frac{r_{1}}{K a_{1}}\right)\right)$ is a stable node or focus, which attracts all orbits of (7) and (8) in the interior of the $S x_{1}$-plane and of the $S x_{2}$ plane, respectively;

(ii) If $\frac{r_{1}}{a_{1}}=\frac{r_{2}}{a_{2}}$ and $r_{3} K \leq \frac{r_{1}}{a_{1}}$, then system (7) and system (8) has two equilibria at $(0,0)$ and $\left(r_{3} K, 0\right)$, where $(0,0)$ is saddle, and $\left(r_{3} K, 0\right)$ is a stable node, which attracts all orbits of (7) and (8) in the interior of the $S x_{1}$-plane and of the $S x_{2}$-plane, respectively;

Summarizing Theorem 5 and Proposition 6 we obtain:

Theorem 7. The following two statements hold.

(i) If $\frac{r_{1}}{a_{1}}=\frac{r_{2}}{a_{2}}$ and $r_{3} K>\frac{r_{1}}{a_{1}}$, then system (1) has infinitely many positive equilibria $E_{+}\left(S, x_{1}, x_{2}\right)$ filling up a segment $L$ which attract all solutions of system (1) with positive initial conditions, the endpoints of $L$ attract all solutions of system (1) with nonnegative initial conditions $\left(S, x_{1}, 0\right)$ or $\left(S, 0, x_{2}\right)$, respectively, and the origin $(0,0,0)$ attracts all solutions of system (1) with nonnegative initial conditions $\left(0, x_{1}, x_{2}\right)$. The phase portrait is shown in Figure 2.

(ii) If $\frac{r_{1}}{a_{1}}=\frac{r_{2}}{a_{2}}$ and $r_{3} K \leq \frac{r_{1}}{a_{1}}$, then system (1) has only two equilibria $(0,0,0)$ and $\left(r_{3} K-\frac{r_{1}}{a_{1}}, 0,0\right)$, the equilibrium $\left(r_{3} K-\frac{r_{1}}{a_{1}}, 0,0\right)$ attracts all solutions of system (1) except the orbits in the $x_{1} x_{2}$-plane, and the equilibrium $(0,0,0)$ attracts all solutions of system (1) in the $x_{1} x_{2}$-plane. The phase portrait is sketched in Figure 3.

Theorem 7 makes sense biologically, which reveals that two competing predators either coexist or co-extinct if they have the same ratio of death rate and propagation rate. When the ratio of death rate and propagation rate of the predator is less than the product of the birth rate and the carrying capacity of the prey, two competing predators coexist at a positive equilibrium. However, if the ratio of death rate and of the propagation rate of the predator is more large than the product of the birth rate and the carrying capacity of the prey, then the two competing predators 


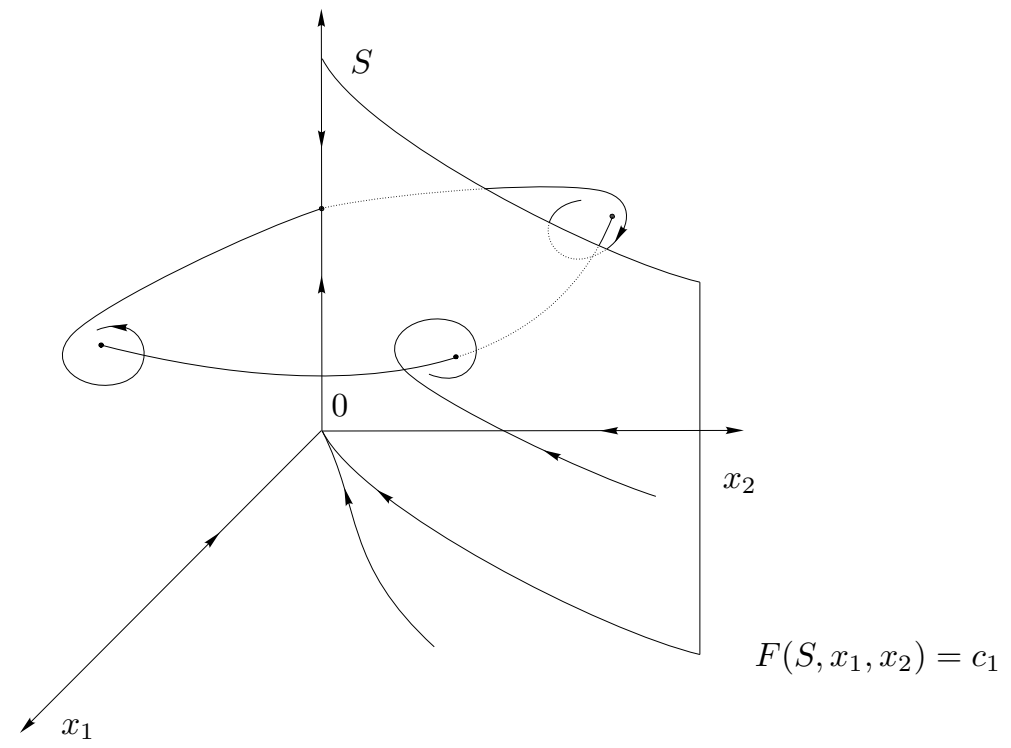

FIGURE 2. Global dynamics of system (1) when $\frac{r_{1}}{a_{1}}=\frac{r_{2}}{a_{2}}$ and $r_{3} K>\frac{r_{1}}{a_{1}}$.

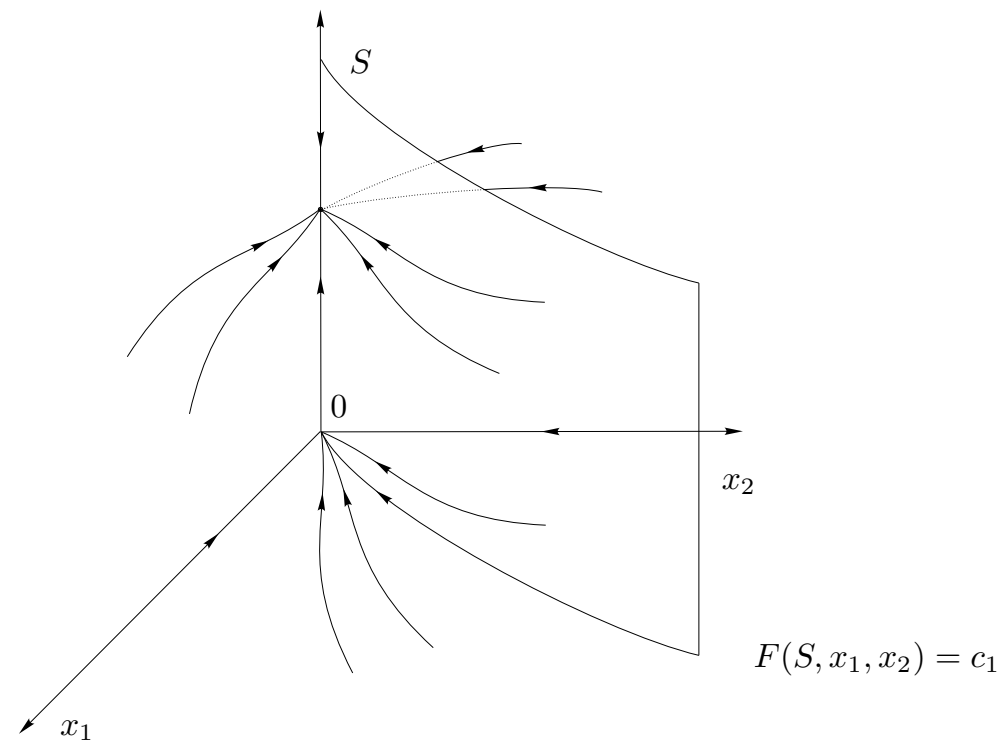

Figure 3. Global dynamics of system (1) when $\frac{r_{1}}{a_{1}}=\frac{r_{2}}{a_{2}}$ and $r_{3} K \leq \frac{r_{1}}{a_{1}}$.

species eventually become extinct even though prey persists. This coincides with our intuition but conflicts with the principle of competitive exclusion. 
In what follows we consider if there exists some values of parameters such that the principle of competitive exclusion holds for system (1). We first give the dynamics of system (1) in $\operatorname{Int} \mathbb{R}_{+}^{3}$.

Theorem 8. If $\frac{r_{1}}{a_{1}} \neq \frac{r_{2}}{a_{2}}$, then the principle of competitive exclusion may hold for system (1). More precisely,

(i) if $\frac{r_{1}}{a_{1}}>\frac{r_{2}}{a_{2}}$, then all orbits of system (1) in $\operatorname{Int}\left(\mathbb{R}_{+}{ }^{3}\right)$ are asymptotic to the orbits on the $S x_{2}$-plane in forward time;

(ii) if $\frac{r_{1}}{a_{1}}<\frac{r_{2}}{a_{2}}$, then all orbits of system (1) in $\operatorname{Int}\left(\mathbb{R}_{+}{ }^{3}\right)$ are asymptotic to the orbits on the $S x_{1}$-plane in forward time.

Proof. Let the set $\mathcal{D}$ be the intersection of the Poincaré compactification ball $B$ and $\mathbb{R}_{+}^{3}$, that is $\mathcal{D}=B \cap \mathbb{R}_{+}^{3}$.

We choose a sufficient large compact set $D_{1}, D_{1} \subset \mathcal{D}$, such that $D_{1}$ does not include $x_{2}=0$, and we choose a sufficient large compact set $D_{2}, D_{2} \subset \mathcal{D}$, such that $D_{2}$ does not include $x_{1}=0$.

From Theorem 4 we know that $D_{1}$ and $D_{2}$ are positive invariant sets of system $(1)$, that is, any solutions $\left(S(t), x_{1}(t), x_{2}(t)\right)$ of system $(1)$ with the initial point $\left(S\left(t_{0}\right), x_{1}\left(t_{0}\right), x_{2}\left(t_{0}\right)\right)$ in $D_{1}$ or in $D_{2}$ will stay in $D_{1}$ or in $D_{2}$, respectively, for all $t \geq t_{0}$.

To prove statement (i) we consider the function

$$
V_{1}\left(S, x_{1}, x_{2}\right)=x_{1}^{a_{2}} x_{2}^{-a_{1}} .
$$

Clearly, $V_{1}\left(S, x_{1}, x_{2}\right)$ is a continuous differential function in $D_{1}$. And

$$
\left.\frac{d V_{1}\left(S, x_{1}, x_{2}\right)}{d t}\right|_{(1)}=a_{1} a_{2}\left(\frac{r_{2}}{a_{2}}-\frac{r_{1}}{a_{1}}\right) V_{1}\left(S, x_{1}, x_{2}\right) \leq 0,
$$

because $\frac{r_{1}}{a_{1}}>\frac{r_{2}}{a_{2}}$. Hence, the solutions $\left(S(t), x_{1}(t), x_{2}(t)\right)$ of system (1) with the initial point $\left(S\left(t_{0}\right), x_{1}\left(t_{0}\right), x_{2}\left(t_{0}\right)\right)$ in $D_{1}$ satisfies

$$
x_{1}^{a_{2}}(t) x_{2}^{-a_{1}}(t)=x_{1}^{a_{2}}\left(t_{0}\right) x_{2}^{-a_{1}}\left(t_{0}\right) e^{a_{1} a_{2} t\left(\frac{r_{2}}{a_{2}}-\frac{r_{1}}{a_{1}}\right)} .
$$

Let

$$
D_{10}=\left\{\left(S, x_{1}, x_{2}\right):\left.\frac{d V_{1}\left(S, x_{1}, x_{2}\right)}{d t}\right|_{(1)}=0,\left(S, x_{1}, x_{2}\right) \in D_{1}\right\} .
$$

Then

$$
D_{10}=\left\{\left(S, x_{1}, x_{2}\right): x_{1}=0\right\},
$$

which is an invariant set of system (1). By LaSalle Principle, we know that all solutions of system (1) in $D_{1}$ are asymptotic to the orbits on the $S x_{2}$-plane in forward time, which leads to statement (i).

Using similar arguments we can obtain statement (ii) by choosing the function

$$
V_{2}\left(S, x_{1}, x_{2}\right)=x_{1}^{-a_{2}} x_{2}^{a_{1}}
$$

in $D_{2}$. This ends the proof.

When $\frac{r_{1}}{a_{1}} \neq \frac{r_{2}}{a_{2}}$, almost all orbits of system (1) are asymptotic to some orbits on the $S x_{1}$-plane or on the $S x_{2}$-plane in forward time. But we do not know what kinds of orbits on the $S x_{1}$-plane or on the $S x_{2}$-plane are the asymptotic ones. To characterize the principle of competitive exclusion holding for system (1), we have 
to show that one of two competing predators must be extinct and the other predator must be survive. So we consider the dynamics of system (1) on the invariant $S x_{1}$ plane and on the invariant $S x_{2}$-plane, respectively.

From Proposition 2 and the knowledge of the 2-dimensional Lotka-Volterra system, we have

Proposition 9. If $\frac{r_{1}}{a_{1}} \neq \frac{r_{2}}{a_{2}}$, then the following statements hold.

(i) If either $r_{3} K>\frac{r_{1}}{a_{1}}>\frac{r_{2}}{a_{2}}$, or $r_{3} K>\frac{r_{2}}{a_{2}}>\frac{r_{1}}{a_{1}}$, then system (7) (or (8)) has three equilibria at $(0,0),\left(r_{3} K, 0\right)$ and $\left(\frac{r_{1}}{a_{1}}, \frac{1}{b_{1}}\left(r_{3}-\frac{r_{1}}{K a_{1}}\right)\right)\left(\operatorname{or}\left(\frac{r_{2}}{a_{2}}, \frac{1}{b_{2}}\left(r_{3}-\right.\right.\right.$ $\left.\left.\frac{r_{2}}{K a_{2}}\right)\right)$, respectively), where $(0,0)$ and $\left(r_{3} K, 0\right)$ are saddles, and $\left(\frac{r_{1}}{a_{1}}, \frac{1}{b_{1}}\left(r_{3}-\right.\right.$ $\left.\left.\frac{r_{1}}{K a_{1}}\right)\right)\left(\left(\frac{r_{2}}{a_{2}}, \frac{1}{b_{2}}\left(r_{3}-\frac{r_{2}}{K a_{2}}\right)\right)\right.$, respectively) is a stable node or focus, which attracts all orbits of $(7)\left((8)\right.$, respectively) in the interior of the Sx$x_{1}$-plane (S $x_{2}$-plane, respectively).

(ii) If $\frac{r_{2}}{a_{2}}<r_{3} K \leq \frac{r_{1}}{a_{1}}$, then system (7) has two equilibria at $(0,0)$ and $\left(r_{3} K, 0\right)$, where $(0,0)$ is a saddle and $\left(r_{3} K, 0\right)$ is a stable node, which attracts all orbits of (7) in the interior of the Sx$x_{1}$-plane. And system (8) has three equilibria at $(0,0),\left(r_{3} K, 0\right)$ and $\left(\frac{r_{2}}{a_{2}}, \frac{1}{b_{2}}\left(r_{3}-\frac{r_{2}}{K a_{2}}\right)\right)$, where $(0,0)$ and $\left(r_{3} K, 0\right)$ are saddles, and $\left(\frac{r_{2}}{a_{2}}, \frac{1}{b_{2}}\left(r_{3}-\frac{r_{2}}{K a_{2}}\right)\right)$ is a stable node or focus, which attracts all orbits of (8) in the interior of the $S x_{2}$-plane, respectively.

(iii) If $\frac{r_{1}}{a_{1}}<r_{3} K \leq \frac{r_{2}}{a_{2}}$, then system (8) has two equilibria at $(0,0)$ and $\left(r_{3} K, 0\right)$, where $(0,0)$ is a saddle and $\left(r_{3} K, 0\right)$ is a stable node, which attracts all orbits of (8) in the interior of the $S x_{2}$-plane. And system (7) has three equilibria at $(0,0),\left(r_{3} K, 0\right)$ and $\left(\frac{r_{1}}{a_{1}}, \frac{1}{b_{1}}\left(r_{3}-\frac{r_{1}}{K a_{1}}\right)\right)$, where $(0,0)$ and $\left(r_{3} K, 0\right)$ are saddles, and $\left(\frac{r_{1}}{a_{1}}, \frac{1}{b_{1}}\left(r_{3}-\frac{r_{1}}{K a_{1}}\right)\right)$ is a stable node or focus, which attracts all orbits of (7) in the interior of the $S x_{1}$-plane, respectively.

(iv) If either $r_{3} K \leq \frac{r_{2}}{a_{2}}<\frac{r_{1}}{a_{1}}$ or $r_{3} K \leq \frac{r_{1}}{a_{1}}<\frac{r_{2}}{a_{2}}$, then system (7) and system (8) has two equilibria at $(0,0)$ and $\left(r_{3} K, 0\right)$, where $(0,0)$ is a saddle, and $\left(r_{3} K, 0\right)$ is a stable node, which attracts all orbits of (7) and (8) in the interior of the $S x_{1}$-plane and of the $S x_{2}$-plane, respectively;

To summarize Theorem 8 and Proposition 9, we give the sufficient and necessary conditions to guarantee that the principle of competitive exclusion holds for system (1) as follows.

Theorem 10. The principle of competitive exclusion holds for system (1) if and only if one of the following conditions holds.

(i) $r_{3} K>\frac{r_{1}}{a_{1}}>\frac{r_{2}}{a_{2}}$. In this case the predator species $x_{1}(t)$ goes to extinction and the predator species $x_{2}(t)$ survives.

(ii) $\frac{r_{2}}{a_{2}}<r_{3} K \leq \frac{r_{1}}{a_{1}}$. In this case the predator species $x_{1}(t)$ goes to extinction and the predator species $x_{2}(t)$ survives.

(iii) $r_{3} K>\frac{r_{2}}{a_{2}}>\frac{r_{1}}{a_{1}}$. In this case the predator species $x_{2}(t)$ goes to extinction and the predator species $x_{1}(t)$ survives.

(iv) $\frac{r_{1}}{a_{1}}<r_{3} K \leq \frac{r_{2}}{a_{2}}$. In this case the predator species $x_{2}(t)$ goes to extinction and the predator species $x_{1}(t)$ survives.

The principle of competitive exclusion does not hold for system (1) if either $r_{3} K \leq$ $\frac{r_{2}}{a_{2}}<\frac{r_{1}}{a_{1}}$, or $r_{3} K \leq \frac{r_{1}}{a_{1}}<\frac{r_{2}}{a_{2}}$. In this case both two competing predators go to 
extinction, and all orbits of system (1) are asymptotic to the equilibrium $\left(r_{3} K, 0,0\right)$ except the orbits on the $x_{1} x_{2}$-plane.

\section{Global DynAmics of SYSTEM (1) With UNLIMited RESOURCE FOR PREY}

In the section we study system (1) with $K=+\infty$ in $\mathbb{R}_{+}^{3}$, i.e. the system

$$
\begin{aligned}
& \frac{d S(t)}{d t}=S(t)\left(r_{3}-b_{1} x_{1}(t)-b_{2} x_{2}(t)\right), \\
& \frac{d x_{1}(t)}{d t}=x_{1}(t)\left(-r_{1}+a_{1} S(t)\right) \\
& \frac{d x_{2}(t)}{d t}=x_{2}(t)\left(-r_{2}+a_{2} S(t)\right) .
\end{aligned}
$$

In a similar way to section 2 we first give the existence and topological classification of the finite equilibria and the equilibria at infinity for system (9), then we study the global dynamics of this system.

3.1. The existence and topological classification of equilibria. It is clear that system (9) has three equilibria the origin $O(0,0,0), \hat{E}_{1}\left(\frac{r_{1}}{a_{1}}, \frac{r_{3}}{b_{1}}, 0\right)$ and $\hat{E}_{2}\left(\frac{r_{2}}{a_{2}}, 0, \frac{r_{3}}{b_{2}}\right)$ in $\mathbb{R}_{+}^{3}$ for all the values of the parameters. Except these equilibria, system (9) may have a positive equilibrium in $\mathbb{R}_{+}^{3}$ for some values of parameters. Doing easy algebra calculations we obtain the following result.

Proposition 11. System (9) has a positive equilibrium $\hat{E}_{+}\left(S, x_{1}, x_{2}\right)$ in $\operatorname{Int}\left(\mathbb{R}_{+}^{3}\right)$ if and only if $\frac{r_{1}}{a_{1}}=\frac{r_{2}}{a_{2}}$. And if system (9) has a positive equilibrium in $\operatorname{Int}\left(\mathbb{R}_{+}^{3}\right)$, then system (9) has infinitely many positive equilibria $\hat{E}_{+}\left(S, x_{1}, x_{2}\right)$ in $\operatorname{Int}\left(\mathbb{R}_{+}^{3}\right)$, which fill up a segment $L_{0}$ with endpoints $\hat{E}_{1}$ and $\hat{E}_{2}$, where

$$
L_{0}=\left\{\left(S, x_{1}, x_{2}\right): S=\frac{r_{1}}{a_{1}}, b_{1} x_{1}+b_{2} x_{2}=r_{3}, x_{1} \geq 0, x_{2} \geq 0\right\} .
$$

We now study the local phase portraits of the boundary equilibria and positive equilibria. Since the Jacobian matrix $J_{1}\left(S, x_{1}, x_{2}\right)$ of system $(9)$ at an equilibrium $\left(S, x_{1}, x_{2}\right)$ is

$$
J_{1}\left(S, x_{1}, x_{2}\right)=\left(\begin{array}{ccc}
r_{3}-b_{1} x_{1}-b_{2} x_{2} & -b_{1} S & -b_{2} S \\
a_{1} x_{1} & -r_{1}+a_{1} S & 0 \\
a_{2} x_{2} & 0 & -r_{2}+a_{2} S
\end{array}\right),
$$

we obtain the following local phase portraits for the equilibria after some computations and the applications of the standard results.

Proposition 12. The following statements hold.

(i) $O(0,0,0)$ is a saddle with a 2 -dimensional stable manifold and an 1-dimensional unstable manifold for all parameters.

(ii) $\hat{E}_{1}\left(\frac{r_{1}}{a_{1}}, \frac{r_{3}}{b_{1}}, 0\right)$ has the following local phase portraits depending on the values of the parameters.

(ii.a) $\hat{E}_{1}$ is a non-hyperbolic equilibrium with an 1-dimensional stable manifold and a 2-dimensional center manifold if $\frac{r_{1}}{a_{1}}<\frac{r_{2}}{a_{2}}$;

(ii.b) $\hat{E}_{1}$ is a zero-Hopf equilibrium with a 3-dimensional center manifold if $\frac{r_{1}}{a_{1}}=\frac{r_{2}}{a_{2}}$ 
(ii.c) $\hat{E}_{1}$ is a non-hyperbolic equilibrium with an 1-dimensional unstable manifold and a 2-dimensional center manifold if $\frac{r_{1}}{a_{1}}>\frac{r_{2}}{a_{2}}$.

(iii) $\hat{E}_{2}\left(\frac{r_{2}}{a_{2}}, 0, \frac{r_{3}}{b_{2}}\right)$ has the following local phase portraits depending on the values of the parameters.

(iii.a) $\hat{E}_{2}$ is a non-hyperbolic equilibrium with an 1-dimensional unstable manifold and a 2-dimensional center manifold if $\frac{r_{1}}{a_{1}}<\frac{r_{2}}{a_{2}}$;

(iii.b) $\hat{E}_{2}$ is a zero-Hopf equilibrium with a 3-dimensional center manifold if $\frac{r_{1}}{a_{1}}=\frac{r_{2}}{a_{2}}$

(iii.c) $\hat{E}_{2}$ is a non-hyperbolic equilibrium with an 1-dimensional stable manifold and a 2-dimensional center manifold if $\frac{r_{1}}{a_{1}}>\frac{r_{2}}{a_{2}}$.

(iv) Each positive equilibrium $\hat{E}_{+}$is a zero-Hopf equilibrium with 3-dimensional center manifold.

It is clear that system (9) has three invariant planes $S=0, x_{1}=0$ and $x_{2}=0$, respectively. Our objective is to study the global dynamics of system $(9)$ in $\mathbb{R}_{+}^{3}$. However, it is not clear whether or not all solutions of system (9) with positive initial conditions are bounded in forward time. So we must study the equilibria of system (9) at infinity. Using the Poincaré compactification of $\mathbb{R}_{+}^{3}$ and similar arguments to those of section 2 , we obtain the following result

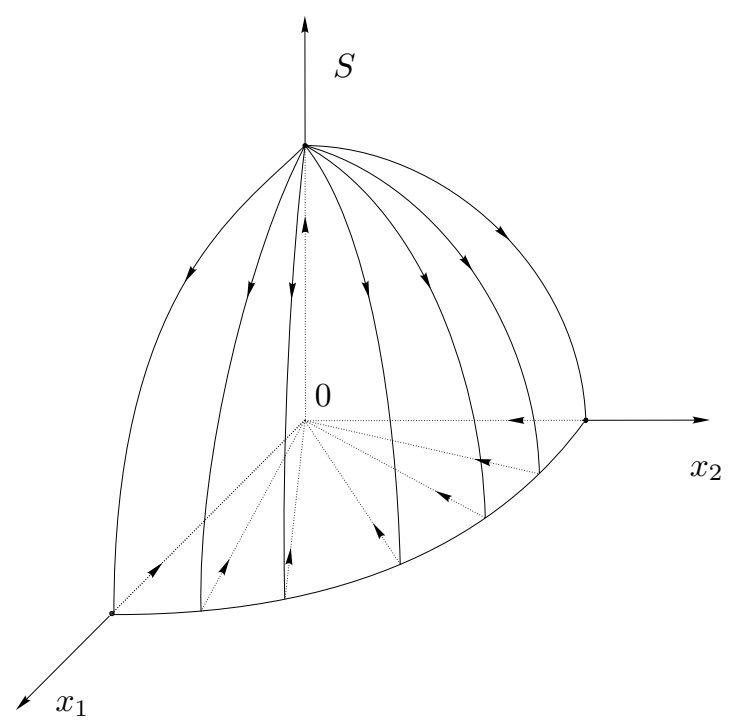

FIGURE 4. The phase portrait of system (9) near all equilibria at infinity on $\mathbb{S}^{2}$.

Proposition 13. The following two statements hold.

(i) System (9) restricted to the compactification of the octant $\mathbb{R}_{+}^{3}$ has an isolated equilibrium $\hat{O}_{i}$ at infinity which is the endpoint of the invariant positive $S$-axis, and has a line filled of equilibria $\hat{E}_{I}$ at infinity which is the end of 
the invariant plane $S=0$ intersection with the compactification of the octant $\mathbb{R}_{+}^{3}$. This line of equilibria fills the quarter of the maximal circle at infinity with the endpoints of the invariant positive $x_{1}$-axis and $x_{2}$-axis.

(ii) Furthermore, $\hat{O}_{i}$ is degenerate with two dimensional unstable manifolds and one dimensional center manifold living in the invariant $S$-axis, and each degenerate equilibria $\hat{E}_{I}$ has 1-dimensional stable manifold living at infinity and two 1-dimensional unstable ones living in the invariant plane $S=0$, which is the central manifold of the point $\hat{E}_{I}$. Moreover, the behavior of the flow near these two 1-dimensional manifolds in the finite positive octant is like a hyperbolic sector. The phase portrait of these equilibria on $\mathbb{S}^{2}$ is shown in Figure 4 .

According to Proposition 13 we get the next result.

Theorem 14. All orbits of system (9) with positive initial conditions are positive and bounded.

3.2. Global dynamics of system (9). From Proposition 11, we know that system (9) has positive equilibria if and only if $\frac{r_{1}}{a_{1}}=\frac{r_{2}}{a_{2}}$. Thus, we discuss the global dynamics of system (9) depending on the relation between of $\frac{r_{1}}{a_{1}}$ and $\frac{r_{2}}{a_{2}}$.

Theorem 15. The following statements hold for the solutions of system (9) with positive initial values.

(i) If $\frac{r_{1}}{a_{1}}>\frac{r_{2}}{a_{2}}$, then all orbits of system (9) in $\operatorname{Int}\left(\mathbb{R}_{+}{ }^{3}\right)$ are asymptotic to some orbits on the $S x_{2}$-plane in forward time, and all orbits on the $S x_{2}$-plane are closed orbits except the equilibrium $\hat{E}_{2}\left(\frac{r_{2}}{a_{2}}, 0, \frac{r_{3}}{b_{2}}\right)$. The phase portrait of system (9) in $\operatorname{Int}\left(\mathbb{R}_{+}{ }^{3}\right)$ is shown in Figure 5 .

(ii) If $\frac{r_{1}}{a_{1}}=\frac{r_{2}}{a_{2}}$, then system (9) in Int $\mathbb{R}_{+}^{3}$ has two independent first integrals $F\left(S, x_{1}, x_{2}\right)=c_{1}$ and $G\left(S, x_{1}, x_{2}\right)=c_{2}$, which completely determines the dynamics of system (9) in $\operatorname{Int} \mathbb{R}_{+}^{3}$, where $F\left(S, x_{1}, x_{2}\right)=x_{1}^{a_{2}} x_{2}^{-a_{1}}$,

$$
G\left(S, x_{1}, x_{2}\right)=\frac{b_{1}}{a_{1}} x_{1}+\frac{b_{2}}{a_{2}} x_{2}+S-\frac{r_{3}}{a_{1}} \ln x_{1}-\frac{r_{1}}{a_{1}} \ln S,
$$

and $c_{1}$ and $c_{2}$ are arbitrary constants. Furthermore, all orbits of system (9) are closed orbits except $\hat{E}_{+}$. The phase portrait of system (9) in $\operatorname{Int}\left(\mathbb{R}_{+}{ }^{3}\right)$ is shown in Figure 6.

(iii) If $\frac{r_{1}}{a_{1}}<\frac{r_{2}}{a_{2}}$, then all orbits of system (9) in $\operatorname{Int}\left(\mathbb{R}_{+}{ }^{3}\right)$ are asymptotic to the orbits on the $S x_{1}$-plane in forward time, and all orbits on the Sx$x_{1}$-plane are closed orbits except the equilibrium $\hat{E}_{1}\left(\frac{r_{1}}{a_{1}}, \frac{r_{3}}{b_{1}}, 0\right)$. The phase portrait of system (9) in $\operatorname{Int}\left(\mathbb{R}_{+}{ }^{3}\right)$ is shown in Figure 7 .

Proof. Using arguments similar to the ones of the proof of Theorem 8, the fact that $\hat{E}_{2}\left(\frac{r_{2}}{a_{2}}, 0, \frac{r_{3}}{b_{2}}\right)$ is a center on the $S x_{2}$-plane, and $\hat{E}_{1}\left(\frac{r_{1}}{a_{1}}, \frac{r_{3}}{b_{1}}, 0\right)$ is a center on the $S x_{1}$-plane, we can obtain statements (i) and (iii). Hence, we only need to prove statement (ii).

Since the function $F\left(S, x_{1}, x_{2}\right)$ satisfies

$$
\left.\frac{d F\left(S, x_{1}, x_{2}\right)}{d t}\right|_{(9)}=a_{1} a_{2}\left(\frac{r_{1}}{a_{1}}-\frac{r_{2}}{a_{2}}\right) F\left(S, x_{1}, x_{2}\right),
$$




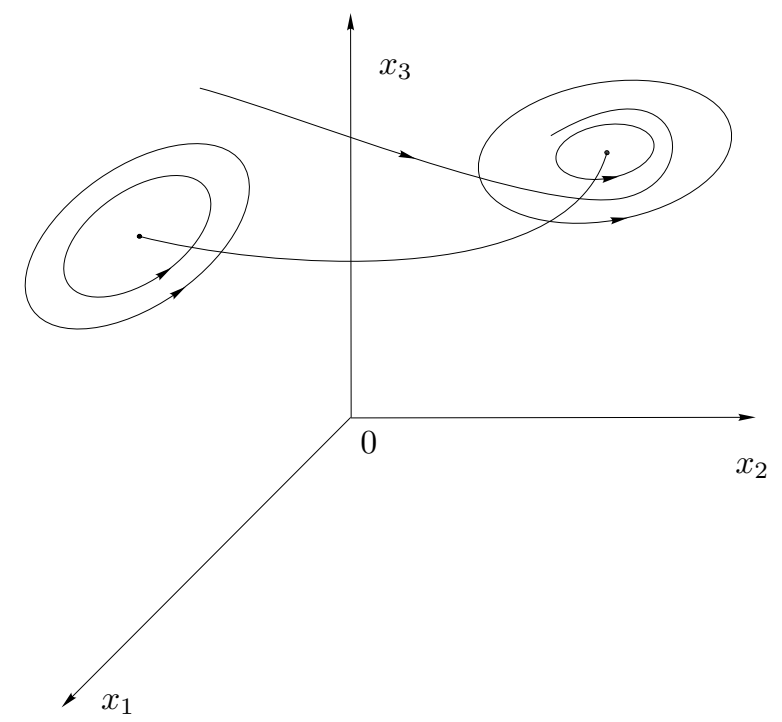

FIGURE 5. Global dynamics of system (9) when $\frac{r_{1}}{a_{1}}>\frac{r_{2}}{a_{2}}$.

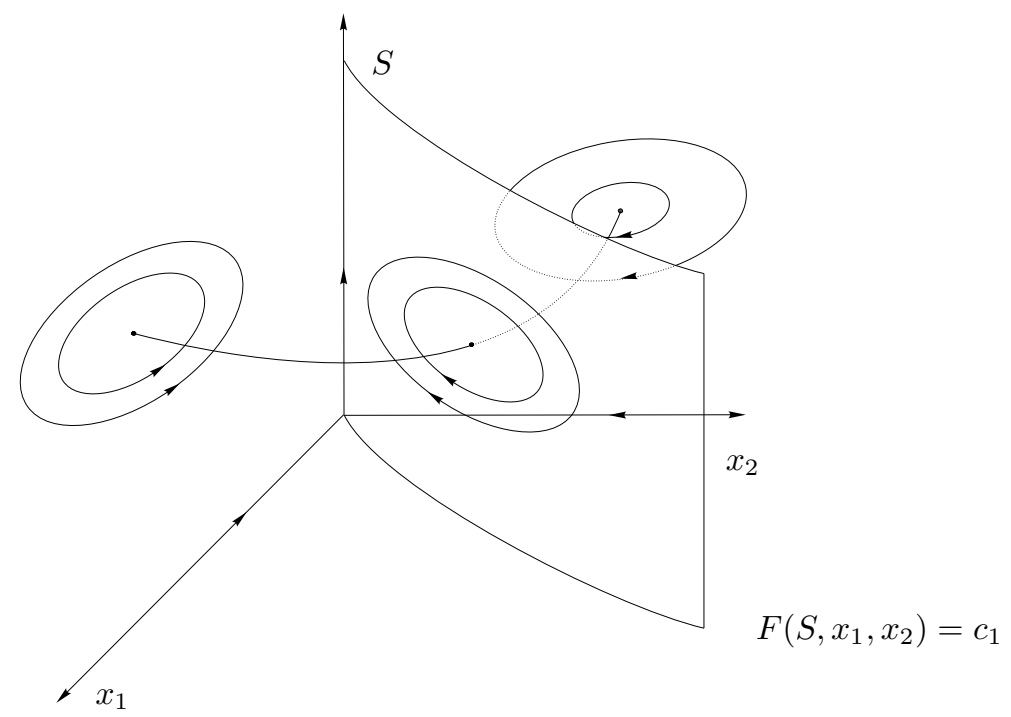

Figure 6. Global dynamics of system (9) when $\frac{r_{1}}{a_{1}}=\frac{r_{2}}{a_{2}}$.

$\left.\frac{d F\left(S, x_{1}, x_{2}\right)}{d t}\right|_{(9)} \equiv 0$ in $\operatorname{Int}\left(\mathbb{R}_{+}{ }^{3}\right)$ if and only if $\frac{r_{1}}{a_{1}}=\frac{r_{2}}{a_{2}}$. Hence, $F\left(S, x_{1}, x_{2}\right)=c_{1}$ is a first integral of system (9) by definition of the first integral, where $c_{1}$ is any a constant.

And the function $G\left(S, x_{1}, x_{2}\right)$ satisfies

$$
\left.\frac{d G\left(S, x_{1}, x_{2}\right)}{d t}\right|_{(9)}=b_{2}\left(\frac{r_{1}}{a_{1}}-\frac{r_{2}}{a_{2}}\right) x_{2},
$$




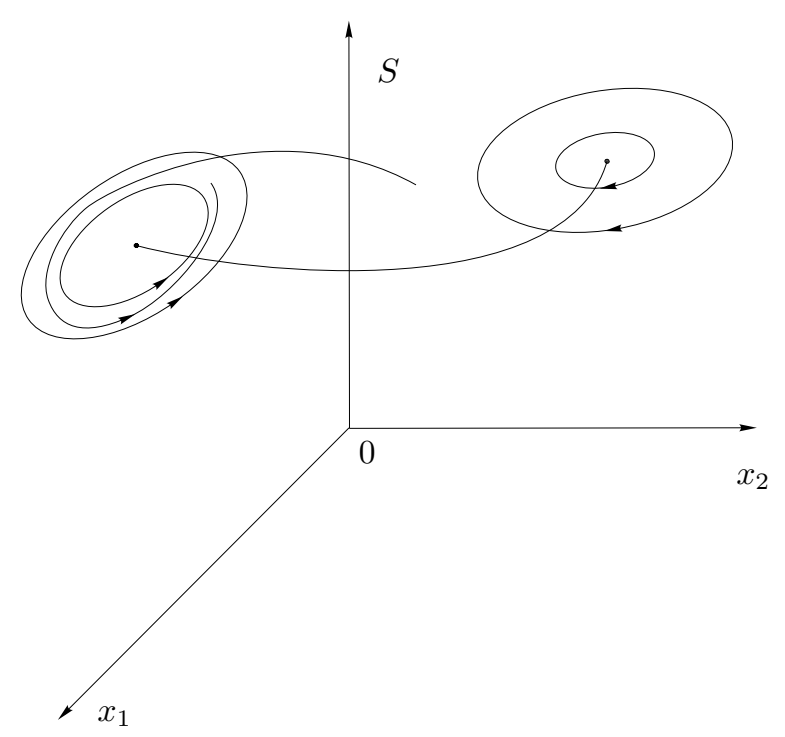

FIGURE 7. Global dynamics of system (9) when $\frac{r_{1}}{a_{1}}<\frac{r_{2}}{a_{2}}$.

$\left.\frac{d G\left(S, x_{1}, x_{2}\right)}{d t}\right|_{(9)} \equiv 0$ in $\operatorname{Int}\left(\mathbb{R}_{+}{ }^{3}\right)$ if and only if $\frac{r_{1}}{a_{1}}=\frac{r_{2}}{a_{2}}$. Hence, $G\left(S, x_{1}, x_{2}\right)=c_{2}$ is the other first integral of system (9) by definition of the first integral, where $c_{2}$ is any a constant.

It can be checked that the two functions, $F\left(S, x_{1}, x_{2}\right)$ and $G\left(S, x_{1}, x_{2}\right)$, are functionally independent. Thus, system (9) has two independent first integrals, which implies that system (9) is completely integrable, and all orbits of system (9) in Int $\mathbb{R}_{+}^{3}$ are the intersection curves of the invariant surface $F\left(S, x_{1}, x_{2}\right)=c_{1}$ and the invariant surface $G\left(S, x_{1}, x_{2}\right)=c_{2}$ in $\operatorname{Int} \mathbb{R}_{+}^{3}$.

Let

$$
F\left(S, x_{1}, x_{2}\right)=c_{1}, c_{1}>0 .
$$

Then the invariant surfaces, $F\left(S, x_{1}, x_{2}\right)=c_{1}$, of system (9) foliate the interior of the first octant (i.e. $\left.\operatorname{Int} \mathbb{R}_{+}^{3}\right)$. On each leaf $F\left(S, x_{1}, x_{2}\right)=c_{1}$, system (9) has a unique positive equilibrium $\hat{E}_{+}\left(\frac{r_{1}}{a_{1}}, x_{1}, x_{2}\right)$ satisfying $x_{1}=\left(c_{1} x_{2}^{a_{1}}\right)^{\frac{1}{a_{2}}}$ and $b_{1} x_{1}+b_{2} x_{2}=r_{3}$. We will demonstrate analytically that each leaf is filled with periodic orbits of system (9) and unique the positive equilibrium $\hat{E}_{+}$in $\operatorname{Int} \mathbb{R}_{+}^{3}$.

Note that the flow of system (9) is invariant on each foliation $F\left(S, x_{1}, x_{2}\right)=c_{1}$ with $c_{1}>0$. Thus, the global dynamics of system (9) in $\operatorname{Int} \mathbb{R}_{+}^{3}$ is equivalent to that of system (9) restricted on each foliation $F\left(S, x_{1}, x_{2}\right)=c_{1}$ for any a $c_{1}>0$.

We now consider the restricted system

$$
\begin{aligned}
& \frac{d S(t)}{d t}=S(t)\left(r_{3}-b_{1}\left(c_{1} x_{2}^{a_{1}}(t)\right)^{\frac{1}{a_{2}}}-b_{2} x_{2}(t)\right), \\
& \frac{d x_{2}(t)}{d t}=x_{2}(t)\left(-r_{2}+a_{2} S(t)\right) .
\end{aligned}
$$


From the above analysis, we know that system (10) has a unique positive equilibrium at $\left(\frac{r_{2}}{a_{2}}, x_{2}^{+}\right)$, where $x_{2}^{+}$is the unique positive solution of

$$
b_{1}\left(c_{1} x_{2}^{a_{1}}\right)^{\frac{1}{a_{2}}}+b_{2} x_{2}=r_{3} .
$$

By computing the Jacobian matrix of system (10) at $\left(\frac{r_{2}}{a_{2}}, x_{2}^{+}\right)$, we have that the equilibrium is a linear center of system (10). And system (10) has a first integral

$$
\frac{b_{1}}{a_{1}}\left(c_{1} x_{2}^{a_{1}}\right)^{\frac{1}{a_{2}}}+\frac{b_{2}}{a_{2}} x_{2}+S-\frac{r_{3}}{a_{1} a_{2}} \ln \left(c_{1} x_{2}^{a_{1}}\right)-\frac{r_{1}}{a_{1}} \ln S=c, c>0 .
$$

Therefore the equilibrium $\left(\frac{r_{2}}{a_{2}}, x_{2}^{+}\right)$is a center of system (10) and all the orbits of system (10) are closed orbits except this equilibrium on the foliation. Because $c_{1}$ is any positive constant, we obtain that all orbits of system (9) are closed orbits except $\hat{E}_{+}$. Hence, the proof is finished.

Remark 16. When $\frac{r_{1}}{a_{1}}=\frac{r_{2}}{a_{2}}$ Theorem 15 shows that system (9) is completely integrable in $\operatorname{Int} \mathbb{R}_{+}^{3}$. But it is not a bi-Hamiltonian system (see [21]).

The global dynamics of system (9) demonstrate that the principle of competitive exclusion holds for system (9) if and only if $\frac{r_{1}}{a_{1}} \neq \frac{r_{2}}{a_{2}}$. And the three species (two predators and a prey) will coexist in the form of periodic oscillation if $\frac{r_{1}}{a_{1}}=\frac{r_{2}}{a_{2}}$.

\section{Discussion}

In this paper we completely analysis the global dynamics of the simplest two predators and a prey Lotka-Volterra model and find that the carrying capacity of the prey $K$ and the ratio of the yield rate and the death rate for predators $\frac{r_{i}}{a_{i}}$, $i=1,2$ play important roles in the global dynamics of this model. This implies some interesting biology results. If the resource for prey is limited (i.e. $K$ is bounded), then there are only three possibilities for two predators species and one prey species: either the principle of competitive exclusion holds, or the coexistence of three species in a stable steady state or the extinction of two predator species and the existence of prey. On the other hand, if the resource for prey is unlimited (i.e. $K$ is unbounded), then there are only two possibilities for two predators species and one prey species: either the principle of competitive exclusion holds, or the coexistence of three species in periodic oscillations. These results lead to a new insight into the mechanism of natural selection and provide a new approach to the problem of biological pest control. Usually biological pest control requires the introduction of a harmless or less harmful predator decreasing the pest predator population to an acceptable level or completely annihilated the pest predator population, which implies the introduced predator species must have a higher survival capacity than the pest predator has in our imagination. Our results show that the intuition is true if the resource for prey is unlimited, and the intuition is not always true if the resource for prey is limited because the two predator species can eventually become extinct and only prey species survives (see statement (ii) of Theorem 7). Hence, this makes sense biologically in pest control.

It is worth to mention that the analysis method in this paper can be applied to $m$ predators and $n$ prey Lotka-Volterra model though our analysis uses the simplest two predators and a prey Lotka-Volterra model. The global dynamics of the $m$ predator and $n$ prey Lotka-Volterra model leaves to be revealed in future. 


\section{ACKNOWLEDGMENTS}

The first author has been supported by the grants MINECO/FEDER MTM 2009-03437, CIRIT 2009SGR, FP7-PEOPLE-2012-IRSES 316338 and 318999, and ICREA Academia. The second author has been partially supported by the National Natural Science Foundations of China (No.10831003 \& No.10925102) and the Program of Shanghai Subject Chief Scientists (No.10XD1406200).

\section{REFERENCES}

1] R. A. Armstrong and R. McGehee, Competitive exclusion, Am. Nat. 115 (1980), 151.

[2] A. Arneodo, P. Coullet And C. Tresser, Occurrence of strange attractors in threedimensional Volterra equations, Phys. Lett. 79A (1980), 259-263.

[3] G. J. Butler and P. Waltman, Bifurcation from a limit cycle in a two predator-one prey ecosystem modeled on a chemostat, J. Math. Biol. 12 (1981), 295-449.

[4] F. Brauer and C. Castillo-Chávez, Mathematical Models in Population Biology and Epidemiology, Springer-Verlag, Heidelberg, 2000.

[5] E. Chauvet; J. E. Paullet; J. P. Previte; Z. Walls, A Lotka-Volterra three-species food chain, Math. Mag. 75 (2002), 243-255.

[6] A. Cima and J. Llibre, Bounded polynomial vector fields, Trans. Amer. Math. Soc. 318 (1990), 557-579.

[7] G. Hardin, The competitive exclusion principle, Science 131 (1960), 1292.

[8] M.W. HiRsch, C.C. Pugh, And M. Shub, Invariant manifolds, Lecture Notes in Mathematics, Vol. 583, Springer-Verlag, Berlin-New York, 1977.

[9] S.-B. Hsu And S. P. Hubbell, Two predators competing for two prey species : Ananalysis of MacArthur's model, Math. Biosci. 47 (1979), 143-171.

[10] S.-B. Hsu, S. P. Hubbell and P. Waltman, Competing predators, SiAM J. Appl. Math 35 (1978), 617-625.

[11] S.-B. Hsu, S. P. Hubbell and P. Waltman, A contribution to the theory of Competing predators, Ecological Monographs 48 (1978), 337-349.

[12] J. P. KEEnER, Oscillatory coexistence in the chemostat: A codimension two unfolding, SIAM J. Appl. Math 43 (1983), 1005-1018.

[13] A. L. KоCH, Competitive coexistence of two predators utilizing the same prey under constant environmental conditions, J. Theoret. Biol. 44 (1974), 373-386.

[14] A. Korobeinikov; G. C. Wake, Global properties of the three-dimensional predator-prey Lotka- Volterra systems, J. Appl. Math. Decis. Sci. 3 (1999), 155-162.

[15] B. Li And H. L. Smith, Global dynamics of microbial competition for two resources with internal storage competition model, J. Math. Biol. 55 (2007), 481-511.

[16] W. Liu, D. Xiao and Y. Yi, Relaxation Oscillations in a class of Predator-prey systems, J. Diff. Eqns. 188 (2003), 306-331.

[17] A. J. LotKA, Elements of Physical Biology Williams and Wilkins, Baltimore, MD, 1925.

[18] M. R. MAY, Limit cycles in predator-prey communities, Science 177 (1972), 900-902.

[19] R. McGehee and R. A. Armstrong, Some mathematical problems concerning the principle of competitive exclusion, J. Diff. Eqs. 23 (1977), 30-52.

[20] S. Muratori and S. Rinald, Remarks on competitive coexistence, SIAM J. Appl. Math. 49 (1989), 1462-1472.

[21] M. Plank, Bi-Hamiltonian systems and Lotka-Volterra equations: a three-dimensional classification, Nonlinearity 9 (1996), 887-896.

[22] H. L. Sмith, Competitive coexistence in an oscillating chemostat, SIAM J. Appl. Math. 40 (1981), 498-522.

[23] H. L. Smith, The interaction of steady state and Hopf bifurcation in a two-predator-one-prey competition model, SIAM J. Appl. Math. 42 (1982), 27-43.

[24] H. L. Smith, P.Waltman, The Theory of the Chemostat, Cambridge University Press, Cambridge, 1995.

[25] V. Volterra, Variazioni e fluttuaziono del numero di individui in specie animali conviventi, Mem. Accad.Lincei, 2 (1926), 31-113. 
[26] G.S.K. Wolkowicz AND Z. Lu, Global dynamics of a mathematical model of competition in the chemostat: general response function and differential death rates, SIAM J. Appl. Math. 52 (1992), 222-245.

Departament de Matemátiques, Universitat Autónoma de Barcelona, 08193 Bellaterra, Barcelona, Catalonia, Spain

E-mail address: jllibre@mat.uab.cat

Department of Mathematics, MOE-LSC, Shanghai Jiao Tong University, Shanghai 200240, CHINA

E-mail address: xiaodm@sjtu.edu.cn 